\title{
Quality Evaluation of Air Material Inventory Management Based on Extenics
}

\section{Theory}

\author{
Chen Zhu ${ }^{1}$, Qian Zhu ${ }^{2}$, Shenyang Liu ${ }^{2}$, Guangxu Luo ${ }^{2}$ \\ ${ }^{1}$ Department of Air Material, Air Force Logistics College, Xige Street, Xuzhou, China \\ ${ }^{2}$ Department of Aviation Four Stations, Air Force Logistics College, Xige Street, Xuzhou, China
}

Keywords: extenics, air material, quality of management, evaluation

\begin{abstract}
Based on the analysis of the influencing factors of the quality management of air material inventory, this paper evaluates the quality of air material inventory management by using the theory of extenics, which solves the problems that the traditional evaluation method cannot reflect the ambiguity and improves the accuracy of the evaluation results. In consequence, the evaluation results are more scientific and reasonable, which is very helpful for solving the problems in the process of inventory management.
\end{abstract}

Inventory management refers to the planning and control activities of the variety and quantity of inventory air materials (Xuejun Chen, 2013). In order to maintain the appropriate storage of air material storage, rational layout, normal circulation, with minimal investment to maximize the demand for air material supply, the best combination of military and economic benefits is achieved. But in the actual management process, frequently there are inaccurate stock book, mismanagement and other reasons, which affect the quality of inventory air materials and result in the formation of dull air material.

This paper evaluates the quality of air material inventory management by using the basic theory of extenics, so as to make an objective and accurate evaluation on the quality of air material inventory management, thus, each unit can make corresponding improvement according to the evaluation result (Xia Zhao,2008). Because the traditional fuzzy evaluation method is subjective and strong, the calculation result is frequently inaccurate, the gray clustering process is complicated, the steps are relatively cumbersome, and the evaluation index value of these methods is a certain value, it cannot reflect the actual situation of ambiguity indicators. However, extension theory can overcome the inherent defects of these methods, so that the evaluation results become more scientific and reasonable (Zhenhua Guo, 2014).

\section{ESTABLISHMENT OF XTENSION MPREHENSIVE EVALUATION MODEL OF INVENTORY ANAGEMENT QUALITY}

\subsection{Establish an evaluation index system}

The air material inventory management quality is affected by many aspects, which not only involve the temperature, humidity, light and other natural environmental factors, but also link with the human factors (Yayu Yin, 2011), the physical and chemical properties of the air material itself are inextricably. There is interaction among the influencing factors.
(1) Development and implementation of air material inventory management system

A scientific management system can guide the air material management, reduce the interference of human factors and the uncertainty in the management process, so that air material management activities are in an orderly manner.

(2) Advantages and disadvantages of air material storage environment

The influence of storage environment on the quality of air material inventory is large, and the factors such as temperature, humidity, light, atmospheric composition and so on may affect the quality of the air material in the reserve process (Yu Chang, 2012).

(3) Integrity degree of warehouse facilities and equipment

Good equipment can avoid damage to the air material and improve the quality of the air material management. The quality of the air material caused by the impact of equipment problems will inevitably affect the daily management of the air material (Wang Chaofeng, 2015).

(4) Use of air material maintenance expenditure

The air material system will use a large amount of expenditure, including the minor maintenance costs of the equipment, the custody of the equipment, the packing, the transportation costs, warehouse water, electricity costs and so on, to supply thousands of air material to units.

(5) Expiration rate of air material storage

As the air material storage time is longer, the physical and chemical properties of the air material will change with the storage time. The strict storage process can make sure that the quality of air material within the specified period of storage does not change, to maintain the integrity of the air material.

(6) Quality inspection of inventory air material

It is necessary to make the managers at all levels grasp the quality status of the inventory air material in time and discover the problems in the process of inventory management, and put forward the rectification measures in time to ensure that the stock air material frequently is new.

(7) Continuous improvement mechanism construction of inventory management 
Air material inventory management is a continuous improvement, sustainable and sophisticated process. With the gradual change of the environment and the continuous improvement of people's understanding level, the existing air material inventory management practices will continue to make improvements (Wenjiao Li, 2016).

(8) Technical level of the air material management personnel

People are the main body of the air material management process. Human business level, ability and quality, operational proficiency and other aspects are likely to affect the quality of the air material management. Such as the low operation level of the air material management personnel may cause quality inspection or maintenance not in place.

(9) Air material management training activities

Air material management training is an important means to improve the technical level of management personnel, management training activities are carried out frequently to improve the business management level, ability quality, operational proficiency, and thus improve the quality of air material management (Guanghong Huang, 2008).

According to the analysis of the above factors, development and implementation of air material inventory management system $c_{1}$, advantages and disadvantages of air material storage environment $c_{2}$, integrity degree of warehouse facilities and equipment $c_{3}$, use of air material maintenance expenditure $c_{4}$, expiration rate of air material storage $c_{5}$, quality inspection of inventory air material $c_{6}$, continuous improvement mechanism construction of inventory management $c_{7}$, technical level of the air material management personnel $c_{8}$, air material management training activities $c_{9}$ nine factors are taken as the assessment indicators of inventory management quality, and the overall goal is the quality of air material inventory management.

According to the actual situation of inventory management, the inventory management quality evaluation results are divided into excellent, good, general, poor four grades, each grade scoring standard as shown in Table 1.

Tab.1 Standard for Evaluation

\begin{tabular}{ccccc}
\hline $\begin{array}{c}\text { management } \\
\text { quality }\end{array}$ & excellent & good & general & poor \\
\hline $\begin{array}{c}\text { scoring } \\
\text { standard }\end{array}$ & $90 \sim 100$ & $70 \sim 89$ & $60 \sim 69$ & $0 \sim 59$ \\
\hline
\end{tabular}

\subsection{Calculation process}

(1) Step 1: Determine the classical domain matter-element matrix

According to the definition of matter-element matrix, in the air material inventory management quality evaluation system, the basic matter-element $R$ refers to the management quality, which contains four levels ( $N=\left\{N_{1}, N_{2}, N_{3}, N_{4}\right\}$ represents excellent, good, general, poor), and $N$ has nine common features
( $c=\left\{c_{1}, c_{2}, \cdots c_{9}\right\}$ respectively represents the assessment indicators of the nine inventory management quality, and the corresponding value is $x=\left\{x_{1}, x_{2}, \cdots x_{9}\right\}$, the range of values shown in Table 1), so

$$
R=(N, C, X)=\left[\begin{array}{ccc}
N & c_{1} & x_{1} \\
& c_{2} & x_{2} \\
& \vdots & \vdots \\
& c_{n} & x_{n}
\end{array}\right]
$$

If the value of the characteristic $c_{i}$ of the $j$ level $N_{j}$ in the four evaluation levels is $X_{i j}$, there is

$$
R_{j}=\left(N_{j}, c_{i}, x_{i j}\right)=\left[\begin{array}{ccc}
N_{j} & c_{1} & x_{1 j} \\
& c_{2} & x_{1 j} \\
& \vdots & \vdots \\
& c_{i} & x_{i j} \\
& \vdots & \vdots \\
& c_{n} & x_{n j}
\end{array}\right], j=\{1,2,3,4\}
$$

So the classical domain matter-element matrix is

$$
\begin{aligned}
R_{o j} & =\left(N_{o j}, C_{o i}, X_{o i j}\right) \\
& =\left[\begin{array}{ccc}
N_{o j} & c_{o 1} & x_{o 1 j} \\
& c_{o 2} & x_{02 j} \\
\vdots & \vdots \\
& c_{o 7} & x_{o 7 j}
\end{array}\right] \\
& =\left[\begin{array}{ccc}
N_{o j} & c_{o 1} & {\left[a_{o 1 j}, b_{o 1 j}\right]} \\
& c_{o 2} & {\left[a_{o 2 j}, b_{o 2 j}\right]} \\
\vdots & \vdots \\
& c_{o 7} & {\left[a_{o 7 j}, b_{o 7 j}\right]}
\end{array}\right]
\end{aligned}
$$

(2) Step 2: Determine the node domain matter-element matrix

The classical domain is summarized and the node domain matter-element matrix $R_{p}$ is obtained:

$$
\begin{aligned}
R_{p} & =\left(N_{p}, C_{p}, X_{p}\right) \\
& =\left[\begin{array}{ccc}
N_{p} & c_{p 1} & x_{p 1} \\
& c_{p 2} & x_{p 2} \\
\vdots & \vdots \\
& c_{p 7} & x_{p 7}
\end{array}\right] \\
& =\left[\begin{array}{ccc}
N_{p} & c_{p 1} & {\left[a_{p 1}, b_{p 1}\right]} \\
& c_{p 2} & {\left[a_{p 2}, b_{p 2}\right]} \\
\vdots & \vdots \\
& c_{p 7} & {\left[a_{p 7}, b_{p 7}\right]}
\end{array}\right]
\end{aligned}
$$

$P$ is the whole of the quality assessment level of the air material inventory management; $x_{p i}$ is the corresponding value of the characteristic $c_{i}$ at the $P$ level, and $x_{p i}=\left\langle a_{p i}, b_{p i}\right\rangle$. 
(3) Step 3: Use the three-scale analytic hierarchy process to calculate the weight of each evaluation index.

As the traditional analytic hierarchy process is influenced by subjective factors, and often do not meet the consistency of the test situation, and the follow-up adjustment is more cumbersome, based on this consideration, in this case, a three-scale analytic hierarchy process is used which does not require consistency checking.

a. Construct a three - scale comparison matrix:

$$
A=\left\lfloor a_{i j}\right\rfloor_{n \times n}
$$

$a_{i j}=\left\{\begin{array}{cc}1 & \mathrm{i} \text { is more important than } \mathrm{j} \\ 0 & \mathrm{i} \text { and } \mathrm{j} \text { are the same important } \\ -1 & \mathrm{j} \text { is more important than } \mathrm{i}\end{array}\right.$

b. Calculate the optimal transfer matrix B of A:

$$
B=\left(b_{i j}\right)_{n \times n}
$$

In formula: $b_{i j}=\frac{1}{n} \sum_{t=1}^{n}\left(a_{i t}+a_{t j}\right)$

c. Calculate the consistency matrix $D$ :

$$
B=\left(b_{i j}\right)_{n \times n}
$$

In formula: $d_{i j}=\exp \left\{b_{i j}\right\}$.

d. Calculate the weight of each indicator:

$$
W_{i}=\frac{\left(\prod_{j=1}^{n} d_{i j}\right)^{\frac{1}{n}}}{\sum_{i=1}^{n}\left(\prod_{j=1}^{n} d_{i j}\right)^{\frac{1}{n}}}
$$

(4) Step 4: Determine the extension relevance of the air material inventory management quality for each level

The extension relevance is the sum of the initial correlation function and its corresponding weight coefficient. Elementary associative function is

$$
k_{j}\left(x_{i}\right)=\left\{\begin{array}{cc}
\frac{\rho\left(x_{i}, x_{\text {oij }}\right)}{\rho\left(x_{i}, x_{\text {pi }}\right)-\rho\left(x_{i}, x_{\text {oij }}\right)} & , \quad \rho\left(x_{i}, x_{\text {pi }}\right)-\rho\left(x_{i}, x_{\text {oij }}\right) \neq 0 \\
100 & , \rho\left(x_{i}, x_{\text {pi }}\right)-\rho\left(x_{i}, x_{\text {oi }}=0, \text { 且 } \rho\left(x_{i}, x_{\text {oij }}\right)>0\right. \\
-100 & , \rho\left(x_{i}, x_{\text {pi }}\right)-\rho\left(x_{i}, x_{\text {oi }}=0 \text {, 且 } \rho\left(x_{i}, x_{\text {oij }}\right)<0\right.
\end{array}\right.
$$

$j=\{1,2,3,4\}, i=\{1,2, \cdots, 9\}$.In formula:

$$
\begin{gathered}
\rho\left(x_{i}, x_{o i j}\right)=\left|x_{i}-\frac{1}{2}\left(a_{o i j}+b_{o i j}\right)\right|-\frac{1}{2}\left(b_{o i j}-a_{o i j}\right) \\
\rho\left(x_{i}, x_{p i}\right)=\left|x_{i}-\frac{1}{2}\left(a_{p i}+b_{p i}\right)\right|-\frac{1}{2}\left(b_{p i}-a_{p i}\right)
\end{gathered}
$$

So the extension relevance is

$$
K_{j}\left(P_{o}\right)=\sum_{i=1}^{9} \omega_{i} k_{j}\left(x_{i}\right)
$$

$K_{j}\left(P_{o}\right)$ is the extension relevance of the air material inventory management B on the level C.

(5) Step 5: Determine the evaluation level

If $K_{j_{o}}=\max K_{j}\left(P_{o}\right), j=1,2,3,4$, the evaluation level of air material inventory management quality is $j_{o}$.

\section{EXAMPLE ANALYSIS}

The air material inventory management quality of a unit is assessed. On the basis of conducting field research and consulting experts, the score of nine inventory management quality evaluation indicators is gotten $c_{1}=75, c_{2}=88, c_{3}=70, c_{4}=77, c_{5}=60$, $c_{6}=71, c_{7}=66, c_{8}=55, c_{9}=47$. The evaluation results are divided into four grades, excellent (90-100), good (70-89), pass (60-69), fail (0-59).

(1) The classical domain matter-element matrix b excellent $(j=1)$

$$
R_{o 1}=\left(N_{o 1}, C_{o i}, X_{o i 1}\right)=\left[\begin{array}{ccc}
N_{o 1} & c_{1} & \langle 90,100\rangle \\
& c_{2} & \langle 90,100\rangle \\
& c_{3} & \langle 90,100\rangle \\
& c_{4} & \langle 90,100\rangle \\
& c_{5} & \langle 90,100\rangle \\
& c_{6} & \langle 90,100\rangle \\
& c_{7} & \langle 90,100\rangle \\
& c_{8} & \langle 90,100\rangle \\
& c_{9} & \langle 90,100\rangle
\end{array}\right]
$$

$2 \operatorname{good}(j=2)$

$$
R_{o 2}=\left(N_{o 2}, C_{o i}, X_{o i 2}\right)=\left[\begin{array}{rrr}
N_{o 2} & c_{1} & \langle 70,89\rangle \\
& c_{2} & \langle 70,89\rangle \\
& c_{3} & \langle 70,89\rangle \\
& c_{4} & \langle 70,89\rangle \\
& c_{5} & \langle 70,89\rangle \\
& c_{6} & \langle 70,89\rangle \\
& c_{7} & \langle 70,89\rangle \\
& c_{8} & \langle 70,89\rangle \\
& c_{9} & \langle 70,89\rangle
\end{array}\right]
$$

$3 \operatorname{pass}(j=3)$

$$
R_{o 3}=\left(N_{o 3}, C_{o i}, X_{o i 3}\right)=\left[\begin{array}{ccc}
N_{o 3} & c_{1} & \langle 60,69\rangle \\
& c_{2} & \langle 60,69\rangle \\
& c_{3} & \langle 60,69\rangle \\
& c_{4} & \langle 60,69\rangle \\
& c_{5} & \langle 60,69\rangle \\
& c_{6} & \langle 60,69\rangle \\
& c_{7} & \langle 60,69\rangle \\
& c_{8} & \langle 60,69\rangle \\
& c_{9} & \langle 60,69\rangle
\end{array}\right]
$$


4 fail $(j=4)$

$$
R_{o 4}=\left(N_{o 4}, C_{o i}, X_{o i 4}\right)=\left[\begin{array}{ccc}
N_{04} & c_{1} & \langle 0,59\rangle \\
& c_{2} & \langle 0,59\rangle \\
& c_{3} & \langle 0,59\rangle \\
& c_{4} & \langle 0,59\rangle \\
& c_{5} & \langle 0,59\rangle \\
& c_{6} & \langle 0,59\rangle \\
& c_{7} & \langle 0,59\rangle \\
& c_{8} & \langle 0,59\rangle \\
& c_{9} & \langle 0,59\rangle
\end{array}\right]
$$

(2) The node domain matter-element matrix

$$
R_{p}=\left(N_{p}, C_{p}, X_{p}\right)=\left[\begin{array}{ccc}
N_{o 1} & c_{p 1} & \langle 0,100\rangle \\
& c_{p 2} & \langle 0,100\rangle \\
& c_{p 3} & \langle 0,100\rangle \\
& c_{p 4} & \langle 0,100\rangle \\
& c_{p 5} & \langle 0,100\rangle \\
& c_{p 6} & \langle 0,100\rangle \\
& c_{p 7} & \langle 0,100\rangle \\
& c_{p 8} & \langle 0,100\rangle \\
& c_{p 9} & \langle 0,100\rangle
\end{array}\right]
$$

(3) Evaluated matter-element

$$
R_{j}=\left(N_{j}, C_{i}, X_{i}\right)=\left[\begin{array}{ccc}
N_{j} & c_{1} & 75 \\
& c_{2} & 88 \\
& c_{3} & 70 \\
& c_{4} & 77 \\
& c_{5} & 60 \\
& c_{6} & 71 \\
& c_{7} & 66 \\
& c_{8} & 55 \\
& c_{9} & 47
\end{array}\right]
$$

(4) Calculate the weight of each evaluation index Construct a three - scale comparison judgment matrix $A$ :

$$
A=\left[\begin{array}{ccccccccc}
0 & -1 & 0 & -1 & 1 & 1 & 1 & 1 & 1 \\
1 & 0 & 1 & 1 & 1 & 1 & 1 & 1 & 1 \\
0 & 1 & 0 & -1 & 1 & 1 & 1 & 1 & 1 \\
1 & 1 & -1 & 0 & 1 & 1 & -1 & 1 & 1 \\
-1 & 1 & 1 & 1 & 0 & -1 & -1 & -1 & 0 \\
-1 & 1 & 1 & 1 & -1 & 0 & 0 & 1 & 1 \\
-1 & 1 & 1 & -1 & -1 & 0 & 0 & 0 & 1 \\
-1 & 1 & 1 & 1 & -1 & 1 & 0 & 0 & 1 \\
-1 & 1 & 1 & 1 & 0 & 1 & 1 & 1 & 0
\end{array}\right]
$$

According to the formulas (1) and (2), the optimal transfer matrix $B$ and the consistency matrix $D$ are obtained respectively, and the weight of each index is obtained by the formula (3):

$$
\begin{aligned}
\omega= & {[0.1029,0.1794,0.1285,0.1150,} \\
& 0.0660,0.1029,0.0737,0.1029,0.1285]
\end{aligned}
$$

(5) Calculate the extension degree of each level According to formula (4):

$$
\begin{aligned}
& k_{1}\left(x_{1}\right)=-0.3750, k_{1}\left(x_{2}\right)=-0.1429, k_{1}\left(x_{3}\right)=-0.4000, \\
& k_{1}\left(x_{4}\right)=-0.3611, k_{1}\left(x_{5}\right)=-0.4286, k_{1}\left(x_{6}\right)=-0.3958, \\
& k_{1}\left(x_{7}\right)=-0.4137, k_{1}\left(x_{8}\right)=-0.4375, k_{1}\left(x_{9}\right)=-0.4778, \\
& k_{2}\left(x_{1}\right)=0.2500, k_{2}\left(x_{2}\right)=0.0909, k_{2}\left(x_{3}\right)=0.0000, \\
& k_{2}\left(x_{4}\right)=0.4375, k_{2}\left(x_{5}\right)=-0.2000, k_{2}\left(x_{6}\right)=0.0357, \\
& k_{2}\left(x_{7}\right)=-0.1053, k_{2}\left(x_{8}\right)=-0.2500, k_{2}\left(x_{9}\right)=-0.3286, \\
& k_{3}\left(x_{1}\right)=-0.1936, k_{3}\left(x_{2}\right)=-0.6129, k_{3}\left(x_{3}\right)=0.0323, \\
& k_{3}\left(x_{4}\right)=-0.2581, k_{3}\left(x_{5}\right)=0.0000, k_{3}\left(x_{6}\right)=-0.0645, \\
& k_{3}\left(x_{7}\right)=0.0968, k_{3}\left(x_{8}\right)=-0.1000, k_{3}\left(x_{9}\right)=-0.2167, \\
& k_{4}\left(x_{1}\right)=-0.3902, k_{4}\left(x_{2}\right)=-0.7073, k_{4}\left(x_{3}\right)=-0.2683, \\
& k_{4}\left(x_{4}\right)=-0.4390, k_{4}\left(x_{5}\right)=-0.0244, k_{4}\left(x_{6}\right)=-0.2927, \\
& k_{4}\left(x_{7}\right)=-0.1707, k_{4}\left(x_{8}\right)=0.0976, k_{4}\left(x_{9}\right)=0.3429,
\end{aligned}
$$

According to the formula (5), the extension degree can be gotten:

$$
\begin{aligned}
K_{1}\left(P_{o}\right) & =-0.3631, K_{2}\left(P_{o}\right)=0.0071, \\
K_{3}\left(P_{o}\right) & =-0.2013, K_{4}\left(P_{o}\right)=-0.2422
\end{aligned}
$$

Because $K_{2}\left(P_{o}\right)=\max K_{j}\left(P_{o}\right), j=1,2,3,4$, the air material inventory management quality is good. But, elementary relevance degree, which represents the use of maintenance expenditure, the technical level of management and training management, is lower, indicating that the management of the unit in the three indicators has loopholes. Therefore, the unit should strengthen the management system, and strictly enforce the system content, in addition, pay attention to the usual quality of inventory air material inspection, and effectively improve the quality of air material inventory management.

\section{CONCLUSIONS}

There are many types of stock, and the quantity is large, which brings great difficulties to the custody and maintenance work, and the inventory air material management quality directly affects the improvement of the troop combat effectiveness. Therefore, this paper uses the extension theory to evaluate the quality of air material inventory management and overcomes the emergence of the subjective experience phenomenon in the process of quality assessment of traditional air material inventory management. The qualitative analysis and quantitative analysis combined to improve the accuracy and scientific evaluation of the assessment, so that, based on the assessment results, the unit can make the appropriate improvements to prevent that the inventory is not accurate, inspection is not in place and other reasons, which affect the improvement of air material guarantee efficiency.

\section{REFERENCES}

[1] Guanghong Huang, Gang Fang, 2005. System engineering 
method and application, Jinan University Press. Guangzhou, 2nd edition.

[2] Wang Chaofeng. Study on progress in researches on aviation materials and resources management: a review of domestic and foreign literatures[J]. Logistic technology, 2015, 34(2): 47-50.

[3] Wenjiao Li, 2016. Optimization of automobile after-sales spare parts inventory management, Logistic technology. 35(5), pp.95-98.

[4] Xuejun Chen, Feng Zhang, Lianwu Zhang, 2013. Battle storage administration, Fortune Press. China, 2nd edition.

[5] Xia Zhao, 2008. An extention synthesis based method on evaluating the sustainable development level of an industrial park. Chemometrics and Intelligent Laboratory, 7(5), pp.23-30.
[6] Yayu Yin, 2011. Research on sustainable utilization level evaluation and management measures of urban water resource based on extension theory, Journal of South China University of Technology, 3(7), pp.: 95-99

[7] Yu Chang, Shanshan Tong, 2012. Development orientation of aircraft material management, Aeronautical Manufacturing Technology. 10(9), pp.87-89.

[8] Zhenhua Guo, Yinghuang Guo, 2014. Extenics theory and its applications-a new interdiscipline-extenics. Journal of Baoji University of Arts and Sciences (Natural Science). 34(2), pp.74-78. 